\title{
Asymmetries on red giant branch surfaces from CHARA/MIRC optical interferometry
}

\author{
A. Chiavassa ${ }^{1}$, R. Norris ${ }^{2}$, M. Montargès ${ }^{3}$, R. Ligi ${ }^{4}$, L. Fossati ${ }^{5}$, L. Bigot ${ }^{1}$, F. Baron ${ }^{2}$, P. Kervella ${ }^{6,7}$, J. D. Monnier ${ }^{8}$, \\ D. Mourard ${ }^{1}$, N. Nardetto ${ }^{1}$, G. Perrin ${ }^{8}$, G. H. Schaefer ${ }^{9}$, T. A. ten Brummelaar ${ }^{9}$, \\ Z. Magic ${ }^{10,11}$, R. Collet ${ }^{12}$, and M. Asplund ${ }^{13}$
}

1 Université Côte d'Azur, Observatoire de la Côte d'Azur, CNRS, Lagrange, CS 34229, 06304 Nice Cedex 4, France e-mail: andrea.chiavassa@oca.eu

2 CHARA and Department of Physics \& Astronomy, Georgia State University, PO Box 4106, Atlanta, GA 30302-4106, USA

3 Institut de Radioastronomie Millimétrique, 300 rue de la Piscine, 38406 Saint-Martin d'Hères, France

4 Aix-Marseille Université, CNRS, LAM (Laboratoire d'Astrophysique de Marseille) UMR 7326, 13388 Marseille, France

5 Space Research Institute, Austrian Academy of Sciences, Schmiedlstrasse 6, 8042 Graz, Austria

${ }^{6}$ Unidad Mixta Internacional Franco-Chilena de Astronomía (UMI 3386), CNRS/INSU, France \& Departamento de Astronomía, Universidad de Chile, Camino El Observatorio 1515, Las Condes, Santiago, Chile

7 LESIA, Observatoire de Paris, PSL Research University, CNRS UMR 8109, Sorbonne Universités, UPMC,

Université Paris Diderot, Sorbonne Paris Cité, 5 place Jules Janssen, 92195 Meudon, France

8 Department of Astronomy, University of Michigan, 918 Dennison Building, Ann Arbor, MI 48109-1090, USA

9 The CHARA Array of Georgia State University, Mount Wilson, CA 91023, USA

10 Niels Bohr Institute, University of Copenhagen, Juliane Maries Vej 30, 2100 Copenhagen, Denmark

11 Centre for Star and Planet Formation, Natural History Museum of Denmark, University of Copenhagen, Øster Voldgade 5-7, 1350 Copenhagen, Denmark

12 Stellar Astrophysics Centre, Department of Physics and Astronomy, Ny Munkegade 120, Aarhus University, 8000 Aarhus C, Denmark

13 Research School of Astronomy \& Astrophysics, Australian National University, Cotter Road, Weston ACT 2611, Australia

Received 13 January 2017 / Accepted 6 March 2017

\begin{abstract}
Context. Red giant branch (RGB) stars are very bright objects in galaxies and are often used as standard candles. Interferometry is the ideal tool to characterize the dynamics and morphology of their atmospheres.

Aims. We aim at precisely characterising the surface dynamics of a sample of RGB stars.

Methods. We obtained interferometric observations for three RGB stars with the MIRC instrument mounted at the CHARA interferometer. We looked for asymmetries on the stellar surfaces using limb-darkening models.

Results. We measured the apparent diameters of HD $197989(\epsilon$ Cyg $)=4.61 \pm 0.02$ mas, HD $189276(\operatorname{HR} 7633)=2.95 \pm 0.01 \mathrm{mas}$, and HD $161096(\beta \mathrm{Oph})=4.43 \pm 0.01$ mas. We detected departures from the centrosymmetric case for all three stars with the tendency of a greater effect for lower $\log g$ of the sample. We explored the causes of this signal and conclude that a possible explanation to the interferometric signal is the convection-related and/or the magnetic-related surface activity. However, it is necessary to monitor these stars with new observations, possibly coupled with spectroscopy, in order to firmly establish the cause.
\end{abstract}

Key words. techniques: interferometric - infrared: stars - stars: atmospheres - stars: individual: HD 197989 stars: individual: HD 189276 - stars: individual: HD 161096

\section{Introduction}

Red giant branch (RGB) stars have evolved from the main sequence and are powered by hydrogen burning in a thin shell surrounding their helium core. This evolutionary phase precedes the asymptotic giant branch (AGB) and is characterised during the evolved states by more expanded and deformed outer layers. RGB stars are bright candles in galaxies, and the accurate determination of their fundamental parameters and chemical composition is essential for tracing the morphology and the evolution of the Galaxy, for probing distant stellar populations, and for characterising globular clusters. Their masses are typically lower than $\sim 2.0 M_{\odot}$ (Salaris et al. 2002) with $4000 \lesssim T_{\text {eff }} \lesssim 5100 \mathrm{~K}$ depending on metallicity, $1.5 \lesssim \log g \lesssim 3.5$, and $3 \lesssim R_{\star} \lesssim 70 R_{\odot}$ (van Belle et al. 1999; Baines et al. 2010).
Like all late-type stars, red giant atmospheres are made complex by convective motions and turbulent flows. Convection contributes significantly to the transportation of energy from the stellar interior to the outer layer, and in the photosphere, it manifests itself as a granulation pattern characterised by dark intergranular lanes of downflowing cooler plasma and bright areas (the granules) where hot plasma rises (see the review of Nordlund et al. 2009). The granules cause an inhomogeneous stellar surface that changes with time. The granulation potentially acts as an intrinsic noise in stellar parameters, radial velocity, and chemical abundance determinations. In addition to this, a magnetic field may be present, as detected in several RGB stars by Aurière et al. (2015), and this may contribute to a bias in the stellar parameter determination. The characterisation of the 
Table 1. Log of observations.

\begin{tabular}{lcccc}
\hline \hline Date (UT) & Target & $N_{\text {block }}$ & $\begin{array}{c}\text { TTO } \\
(\mathrm{min})\end{array}$ & Calibrators \\
\hline 2016 Jul. 6 & HD 161096 $(\beta$ Oph $)$ & 2 & 20 & $\epsilon$ Ser, 72 Oph, $\lambda$ Aql \\
2016 Jul. 6 & HD 189276 (HR 7633) & 1 & 10 & $\iota$ Cyg, HR 8185 \\
2016 Jul. 6 & HD 197989 $(\epsilon$ Cyg $)$ & 1 & 15 & $\gamma$ Lyr \\
2016 Jul. 7 & $\beta$ Oph & 1 & 25 & $\epsilon$ Ser, 72 Oph \\
2016 Jul. 7 & HR 7633 & 1 & 20 & $\iota$ Cyg, \\
2016 Jul. 7 & $\epsilon$ Cyg & 1 & 30 & 17 Cyg, $\sigma$ Cyg \\
\hline
\end{tabular}

Notes. TTO is the total time observed. Calibrator diameters (mas): $\epsilon$ Ser $=0.689 \pm 0.048(1) ; 72 \mathrm{Oph}=0.684 \pm 0.048(2) ; \lambda \mathrm{Aql}=0.518 \pm$ 0.036 (2); ८ Cyg = $0.586 \pm 0.041$ (2); HR $8185=1.067 \pm 0.076$ (2); $\gamma \mathrm{Lyr}=0.737 \pm 0.015(3) ; 17 \mathrm{Cyg}=0.721 \pm 0.051(2) ; \sigma \mathrm{Cyg}=0.542 \pm$ 0.021 (4). All the diameters, except for $\epsilon$ Ser, are in the $H$ band. $\epsilon$ Ser is given in $K$ band, variation across wavelength for this diameter is extimated to be $\sim 0.01$ mas with negligible effect on the data reduction.

References. (1) Boyajian et al. (2012); (2) Bonneau et al. (2006); (3) Monnier et al. (2012); (4) Zhao et al. (2008).

dynamics and morphology of RGB stars is important to quantify the effect of the granulation and magnetic fields, and thanks to its high angular resolution, interferometry is the ideal tool for this purpose.

In this Letter, we present the detection of an interferometric signal at high spatial frequencies for three RGB stars using the MIRC instrument mounted at the CHARA interferometer. We analyse the possible causes of this signal.

\section{Interferometric observations with MIRC at CHARA}

We collected observations of three stars (Table 1) using the Michigan Infrared Combiner (MIRC) on the Georgia State University Center for High Angular Resolution Astronomy (CHARA). The CHARA array is located on Mount Wilson, CA, and consists of six $1 \mathrm{~m}$ telescopes for a total of 15 baselines ranging in length from $34 \mathrm{~m}$ to $331 \mathrm{~m}$, resulting in an angular resolution of $\sim 0.5$ mas in the $H$ band (ten Brummelaar et al. 2005). The detailed parameters of the RGB stars are reported in Table 2.

The MIRC (Monnier et al. 2004, 2012) is a six-beam combiner operating in the $H$ band $(1.5-1.8 \mu \mathrm{m})$ at low spectral resolution $(R=30)$. Each observing block consists of observations of a calibrator, a target, and when possible, a second calibrator. Time spent collecting data on the target ranged within 10 to $30 \mathrm{~min}$, excluding background and other calibration frames, depending on observing conditions. We used the latest version of the MIRC reduction pipeline (as of October 2016) and as previously described in Monnier et al. (2007) and Monnier et al. (2012). The pipeline uses Fourier transforms to compute squared visibilities, which are then averaged and corrected for biases. We determined the bispectrum using the phases and amplitudes of three baselines in a closed triangle. We calibrated photometric amplitudes using a beam splitter following spatial filtering (Che et al. 2010) $)^{1}$. Because we do not expect significant brightness variation over short time periods for these targets, we combined the two nights of observations for each star into single files, accounting for systematic error by applying multiplicative

\footnotetext{
1 This research has made use of the Jean-Marie Mariotti Center SearchCal service Bonneau et al. (2006), available at http://www. jmmc. fr/searchcal, co-developped by FIZEAU and LAOG/IPAG, and of CDS Astronomical Databases SIMBAD and VIZIER, available at http://cdsweb.u-strasbg.fr/
}
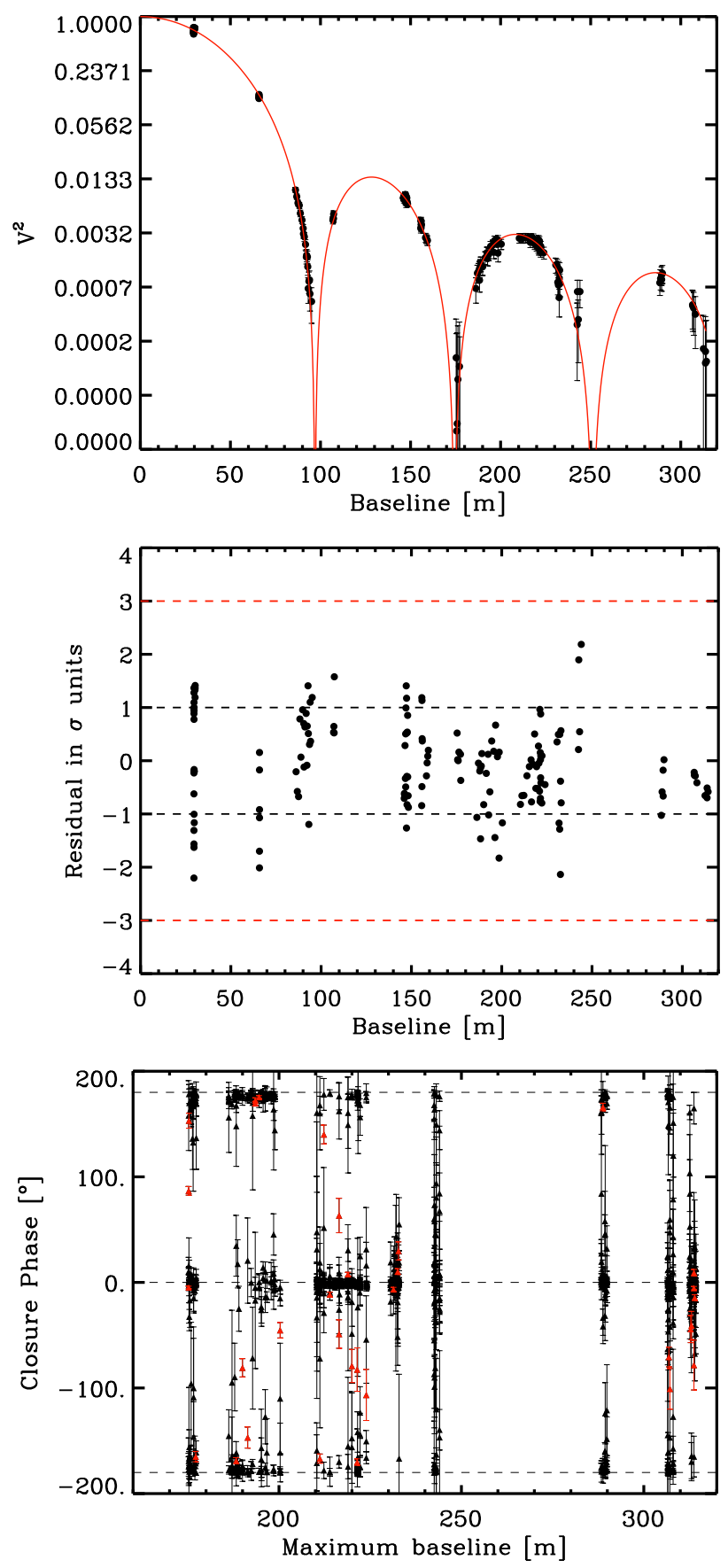

Fig. 1. Top and central panels: squared visibilities with the limbdarkening fit residuals (top and central panels) at the wavelength band $1.6004 \pm 0.0036 \mu \mathrm{m}$. The continuous line in the top panel corresponds to the limb-darkening fit whose parameters are reported in Table 3. The black horizontal dashed lines in the central panel correspond to the value of $1 \sigma$, and the red line shows the value of $3 \sigma$. Bottom panel: closure phase data points of $\beta$ Oph (Table 2) for all the wavelengths. The red data correspond to closure phase departures larger than $3 \sigma$ (see Fig. 2). The horizontal dashed lines in the bottom panel display the zero or $\pm 180^{\circ}$ values.

and additive errors as described in Monnier et al. (2012). At low visibilities $\left(\lesssim 10^{-3}\right)$, the signal-to-noise ratio of the data decreases because of cross-talk. We therefore remain cautious when interpreting data at such low visibilities.

The observations were collected in eight different wavelength bands: $1.7379 \pm 0.0031,1.7055 \pm 0.0033,1.6711 \pm$ $0.0035,1.6361 \pm 0.0035,1.6004 \pm 0.0036,1.5642 \pm 0.0037$, 
Table 2. Parameters of the RGB stars.

\begin{tabular}{cccccccc}
\hline \hline Stars & $\begin{array}{c}\text { Spectral } \\
\text { type }^{a}\end{array}$ & $H$ mag & $\begin{array}{c}M \\
{\left[M_{\odot}\right]}\end{array}$ & {$[\mathrm{Fe} / \mathrm{H}]$} & $\begin{array}{c}T_{\text {eff }} \\
{[\mathrm{K}]}\end{array}$ & $\begin{array}{c}\log g \\
{[\operatorname{cgs}]}\end{array}$ & $\begin{array}{c}R \\
{\left[R_{\odot}\right]}\end{array}$ \\
\hline$\epsilon$ Cyg & K0III-IV & $0.200^{b}$ & $1.84 \pm 0.31^{d}$ & $-0.11 \pm 0.10^{d}$ & $4778 \pm 49^{d}$ & $2.62 \pm 0.10^{d}$ & $11.08 \pm 0.25^{d}$ \\
HR 7633 & K4.5IIIa & $0.919^{b}$ & - & - & $4050^{e}$ & $1.70^{e}$ & - \\
$\beta$ Oph & K2III & $0.354^{c}$ & $1.63 \pm 0.18^{d}$ & $0.13 \pm 0.10^{d}$ & $4520 \pm 44^{d}$ & $2.42 \pm 0.07^{d}$ & $13.13 \pm 0.32^{d}$ \\
\hline
\end{tabular}

References. ${ }^{(a)}$ Gray et al. (2003); ${ }^{(b)}$ Ducati (2002); ${ }^{(c)}$ Laney et al. (2012); ${ }^{(d)}$ Reffert et al. (2015); ${ }^{(e)}$ Lafrasse et al. (2010).
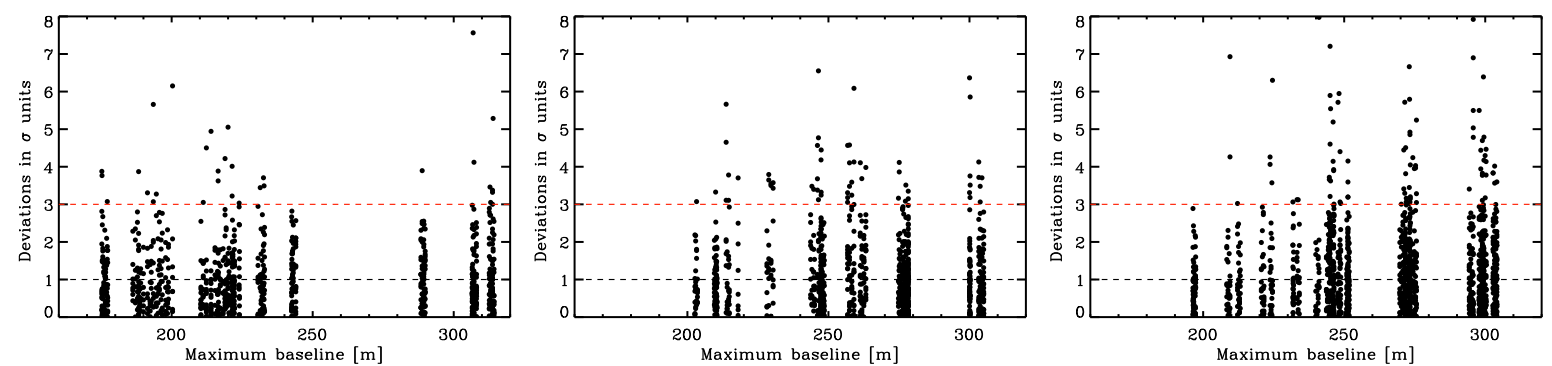

Fig. 2. Closure phase departures from zero or $\pm 180^{\circ}$ for all the observed stars and for all the wavelengths. For every data point, the lowest value between $\mid$ data $-0^{\circ} \mid$, $\mid$ data $-180^{\circ} \mid$, and $\mid$ data $+180^{\circ} \mid$ is computed and normalised by the corresponding observed error, $\sigma . \beta$ Oph is shown in the left panel, $\epsilon$ Cyg in the central, and HR 7633 in the right panel. The horizontal black line corresponds to the value of $1 \sigma$ and the red line to $3 \sigma$.

Table 3. Apparent diameters of the observed stars at $1.6004 \pm$ $0.0036 \mu \mathrm{m}$.

\begin{tabular}{ccccc}
\hline \hline Star & $\begin{array}{c}\text { LD power-law } \\
\text { exponent }\end{array}$ & $\begin{array}{c}\theta_{\mathrm{LD}} \\
{[\mathrm{mas}]}\end{array}$ & $\begin{array}{c}\theta_{\mathrm{LD}} \\
{\left[R_{\odot}\right]}\end{array}$ & $\overline{\chi^{2}}$ \\
\hline$\epsilon$ Cyg & $0.25 \pm 0.01$ & $4.61 \pm 0.02$ & $22.09 \pm 0.15$ & 2.45 \\
HR 7633 & $0.14 \pm 0.01$ & $2.95 \pm 0.01$ & $178.11 \pm 11.11$ & 1.32 \\
$\beta$ Oph & $0.25 \pm 0.01$ & $4.43 \pm 0.01$ & $23.89 \pm 0.16$ & 1.01 \\
\hline
\end{tabular}

$1.5273 \pm 0.0035$, and $1.4833 \pm 0.0033 \mu \mathrm{m}$. In the following, we use the wavelength band centred at $1.6004 \pm 0.0036 \mu \mathrm{m}$ for the apparent diameter determination with visibility curves because it corresponds to the $\mathrm{H}^{-}$continuous opacity minimum (consequently closest to the continuum forming region). For all the closure phase plots, we used the full set of wavelength bands.

\section{Discussion}

We fitted the data, based only on the squared visibilities, with an power-law limb-darkened disk model whose parametric values are reported in Table 3. Figure 1 displays the example of $\beta$ Oph, while the other stars are reported in Figs. A.1 and A.2. The limb-darkening fit is very good (Table 3 ) with larger residuals for HR 7633. We report the first measure of the radius for HR 7633, and while the radius of $\epsilon$ Cyg is in good agreement with Reffert et al. (2015), the radius of $\beta$ Oph is slightly smaller.

The observed closure phases display values different from zero or $\pm 180^{\circ}$ for all the observed stars. To determine the amplitude of these deviations, we selected the lowest value between $\mid$ data $-0^{\circ} \mid$, $\mid$ data $-180^{\circ} \mid$, and $\mid$ data $+180^{\circ} \mid$ for each data point and then normalised it by the corresponding observed error, $\sigma$. We plot the data departures in Fig. 2, which shows several points diverging from the centrosymmetric case for values higher than $3 \sigma$ : the closure phase departures are smaller for $\beta$ Oph (33 points over 920, 3.6\%, higher than $3 \sigma$ ), intermediate for $\epsilon$ Cyg (70 points over 1056, 6.6\%, higher than $3 \sigma$ ), and larger for HR 7633 ( 88 points over 1384, 6.3\%, higher than $3 \sigma$ ). For $\beta$ Oph and $\epsilon \mathrm{Cyg}$, the spatial frequencies spanned extend to the fourth lobe, while for HR 7633, they only reach (partially) the third lobe. The contribution of the small-scale structures increases with the frequency, and that HR 7633 displays departures already on the second lobe indicates that this star is most likely the most asymmetric of the three.

Moreover, the closure phase departures of the three stars seem to be correlated with $\log g$ of the stars (Table 2), the latter is an indicator of the evolutionary phase: the largest deviations are for HR 7633, which has $\log g=1.70$. This denotes that the size of the granules become more significant with respect to the disk size as the surface gravity decreases, and therefore brightness fluctuations become more important. This idea is supported by previous work showing even larger departures from centrosymmetry for very evolved stars such as AGBs (Wittkowski et al. 2016; Chiavassa et al. 2010c; Ragland et al. 2006) and red supergiant stars (Montargès et al. 2016; Chiavassa et al. 2010b). However, we also note that we detected the largest deviations for the faintest star (HR 7633), and this may indicate that we underestimated the errors.

We now present a tentative explanation of these closure phase departures. A more complete analysis will be presented in a forthcoming paper.

Stellar surface asymmetries in the brightness distribution can be either due to convection-related and/or activity-related structures, to a companion, or to a clumpy dust envelope around the stars. In the following, we analyse the different possibilities.

A first hypothesis concerns convection-related surface structures affecting the interferometric observables. The expected convection turnover time in such a star is between hours to days or weeks, depending on the stellar fundamental parameters. Chiavassa et al. (2010a, 2014) showed that stellar granulation manifests itself as surface asymmetries in the brightness distribution, and more precisely, in the closure phase signal.

A second hypothesis is the stellar magnetic activity. Chiavassa et al. (2014) and Ligi et al. (2015) showed that star spots caused by the stellar magnetic field affect the closure phase signal in a similar way as the granulation. To determine its impact, we estimated the indicator $S_{\mathrm{MW}}$ based on the historic stellar activity data of chromospheric line emission. It measures the 
strength of the chromospheric emission core of the $\mathrm{H}$ and $\mathrm{K}$ lines of Ca II (Middelkoop 1982). For the three stars, we converted $S_{\mathrm{MW}}$ into $\log R_{\mathrm{HK}}^{\prime}$ (Table 4) value using the calibration provided by Mittag et al. (2013) because $\log R_{\mathrm{HK}}^{\prime}$ is independent of the stellar type. In particular, we adopted their Eq. (13) and calibration for giant stars (their Eq. (23)), which is valid for stars with $0.76<B-V<1.18$. It should be noted that the $B-V$ colour of HR 7633 lies beyond the range of validity of the calibration, which implies that the activity indicator may not be reliable. The values for $\beta$ Oph and $\epsilon$ Cyg fall into or very close to the stellar inactivity region (Figs. 3 and 4 in Wright 2004). However, the star spots may still exist even if the activity is low, and their signature can only be distinguished by performing more interferometric observations coupled with spectroscopic observations.

The source HR7633 appears to be an active star with $\log R_{\mathrm{HK}}^{\prime}=-4.132$. In addition to this, the stellar diameter of $178.11 R_{\odot}$ of HR 7633 (Table 3 ) as well as its low surface gravity (Table 2) seem to indicate that this star approaches the red giant tip towards the AGB phase. This evolutionary step is characterised by prominent stellar granulation accompanied by nonnegligible dynamics (Ludwig \& Kučinskas 2012).

Concerning any possible companions, only $\epsilon$ Cyg is a known double system (Poveda et al. 1994) where the primary star ( $\epsilon \mathrm{Cyg}$ ) is $\approx 25000$ brighter than its companion (dwarf M4 star) with $m_{\mathrm{V}}=2.45\left(m_{\mathrm{H}}=0.20\right.$, Ducati 2002) and $m_{\mathrm{V}}=11.98$ $\left(m_{\mathrm{H}}=9.58^{2}\right)$, respectively. In addition to this, the two stars lie $78.1^{\prime \prime}$ from each other. For these reasons, we estimate that the secondary star has a negligible effect on the observed data.

No dust production is detected around RGBs for $\beta$ Oph and HR 7633 (McDonald et al. 2011; and McDonald, priv. comm.). The same is expected for $\epsilon$ Cyg. Dust is a sign of strong massloss; in evolved AGB stars, for example, the mass loss can be 1000 greater than in RGB stars.

Stellar convection-related and/or the magnetic-related surface activity are the most plausible explanation. To firmly establish the cause, it is necessary to monitor these stars with new interferometric observations, possibly coupled with spectropolarimetric data in the visible in order to measure their magnetic activity strength.

\section{Conclusions}

We presented observations of three RGB stars using the MIRC instrument at the CHARA interferometer. We showed that for all stars, the limb-darkening fit is very good with larger residuals for HR 7633. We measured the apparent diameters of HD $197989(\epsilon$ Cyg $)=4.61 \pm 0.02$ mas, HD $189276($ HR 7633 $)=$ $2.95 \pm 0.01$ mas, and HD $161096(\beta$ Oph $)=4.43 \pm 0.01$ mas.

Moreover, the closure phases denote departure points from the centrosymmetric case (closure phases not equal to 0 or $\pm 180^{\circ}$ ) with values greater than $3 \sigma$. The departures seem to be qualitatively correlated with $\log g$ of the observed stars. HR 7633, with the lowest $\log g$ of the sample, shows the highest deviations: the more the star evolves, the more significant the size of the granules becomes with respect to the disk size.

We explored the possible causes of the break in symmetry in the brightness distribution and found that a possible explanation of this interferometric signal is the granulation and/or the stellar magnetic activity at its surface. However, it is not possible to confirm this at the moment, and a more complete analysis will be presented in a forthcoming paper.

\footnotetext{
2 Estimate based on PHOENIX models (e.g. Allard et al. 1997, and perso.ens-lyon.fr/france.allard/)
}

Table 4. Photometric colours and stellar activity indicator of the observed RGB stars.

\begin{tabular}{ccccc}
\hline \hline Star & $B-V$ & $\begin{array}{c}\text { Parallax }^{c} \\
{[\mathrm{mas}]}\end{array}$ & $S_{\mathrm{MW}}{ }^{d}$ & $\log R_{\mathrm{HK}}^{\prime}$ \\
\hline$\epsilon$ Cyg & $1.04^{a}$ & $44.86 \pm 0.12$ & 0.10 & -4.910 \\
HR 7633 & $1.55^{a}$ & $3.56 \pm 0.21$ & 0.29 & -4.132 \\
$\beta$ Oph & $1.18^{b}$ & $39.85 \pm 0.17$ & 0.11 & -4.738 \\
\hline
\end{tabular}

References. ${ }^{(a)}$ Ducati (2002); ${ }^{(b)}$ Oja (1993); ${ }^{(c)}$ van Leeuwen (2007); (d) Duncan et al. (1991).

Acknowledgements. This work is based upon observations obtained with the Georgia State University Center for High Angular Resolution Astronomy Array at Mount Wilson Observatory. The CHARA Array is supported by the National Science Foundation under Grants Nos. AST-1211929 and AST-1411654. Institutional support has been provided from the GSU College of Arts and Sciences and the GSU Office of the Vice President for Research and Economic Development. A.C., M.M., P.K., G.P. acknowledge financial support from "Programme National de Physique Stellaire" (PNPS) of CNRS/INSU, France. R.N. acknowledges the Fizeau exchange visitors program in optical interferometry - WP14 OPTICON/FP7 (2013-2016, grant number 312430). R.C. acknowledges the funding provided by The Danish National Research Foundation (Grant DNRF106).

\section{References}

Allard, F., Hauschildt, P. H., Alexander, D. R., \& Starrfield, S. 1997, ARA\&A, 35,137

Aurière, M., Konstantinova-Antova, R., Charbonnel, C., et al. 2015, A\&A, 574, A90

Baines, E. K., Döllinger, M. P., Cusano, F., et al. 2010, ApJ, 710, 1365

Bonneau, D., Clausse, J.-M., Delfosse, X., et al. 2006, A\&A, 456, 789

Boyajian, T. S., McAlister, H. A., van Belle, G., et al. 2012, ApJ, 746, 101

Che, X., Monnier, J. D., \& Webster, S. 2010, in Optical and Infrared Interferometry II, Proc. SPIE, 7734, 77342V

Chiavassa, A., Collet, R., Casagrande, L., \& Asplund, M. 2010a, A\&A, 524, A93

Chiavassa, A., Haubois, X., Young, J. S., et al. 2010b, A\&A, 515, A12

Chiavassa, A., Lacour, S., Millour, F., et al. 2010c, A\&A, 511, A51

Chiavassa, A., Ligi, R., Magic, Z., et al. 2014, A\&A, 567, A115

Ducati, J. R. 2002, VizieR Online Data Catalog: 2/237

Duncan, D. K., Vaughan, A. H., Wilson, O. C., et al. 1991, ApJS, 76, 383

Gray, R. O., Corbally, C. J., Garrison, R. F., McFadden, M. T., \& Robinson, P. E. 2003, AJ, 126, 2048

Lafrasse, S., Mella, G., Bonneau, D., et al. 2010, in Optical and Infrared Interferometry II, Proc. SPIE, 7734, 77344E-77344E-11

Laney, C. D., Joner, M. D., \& Pietrzyński, G. 2012, MNRAS, 419, 1637

Ligi, R., Mourard, D., Lagrange, A.-M., Perraut, K., \& Chiavassa, A. 2015, A\&A, 574, A69

Ludwig, H.-G. \& Kučinskas, A. 2012, A\&A, 547, A118

McDonald, I., Boyer, M. L., van Loon, J. T., et al. 2011, ApJS, 193, 23

Middelkoop, F. 1982, A\&A, 107, 31

Mittag, M., Schmitt, J. H. M. M., \& Schröder, K.-P. 2013, A\&A, 549, A117

Monnier, J. D., Berger, J.-P., Millan-Gabet, R., \& ten Brummelaar, T. A. 2004, in New Frontiers in Stellar Interferometry, ed. W. A. Traub, Proc. SPIE, 5491, 1370

Monnier, J. D., Zhao, M., Pedretti, E., et al. 2007, Science, 317, 342

Monnier, J. D., Che, X., Zhao, M., et al. 2012, ApJ, 761, L3

Montargès, M., Kervella, P., Perrin, G., et al. 2016, A\&A, 588, A130

Nordlund, A., Stein, R. F., \& Asplund, M. 2009, Liv. Rev. Sol. Phys., 6, 2

Oja, T. 1993, A\&AS, 100, 591

Poveda, A., Herrera, M. A., Allen, C., Cordero, G., \& Lavalley, C. 1994, Rev. Mex. Astron. Astrofis., 28, 43

Ragland, S., Traub, W. A., Berger, J.-P., et al. 2006, ApJ, 652, 650

Reffert, S., Bergmann, C., Quirrenbach, A., Trifonov, T., \& Künstler, A. 2015, A\&A, 574, A116

Salaris, M., Cassisi, S., \& Weiss, A. 2002, PASP, 114, 375

ten Brummelaar, T. A., McAlister, H. A., Ridgway, S. T., et al. 2005, ApJ, 628, 453

van Belle, G. T., Lane, B. F., Thompson, R. R., et al. 1999, AJ, 117, 521 van Leeuwen, F. 2007, A\&A, 474, 653

Wittkowski, M., Chiavassa, A., Freytag, B., et al. 2016, A\&A, 587, A12

Wright, J. T. 2004, AJ, 128, 1273

Zhao, M., Gies, D., Monnier, J. D., et al. 2008, ApJ, 684, L95 
A. Chiavassa et al.: Asymmetries on red giant branch surfaces from CHARA/MIRC optical interferometry

\section{Appendix A: Additional figures for $\epsilon$ Cyg} and HR 7633
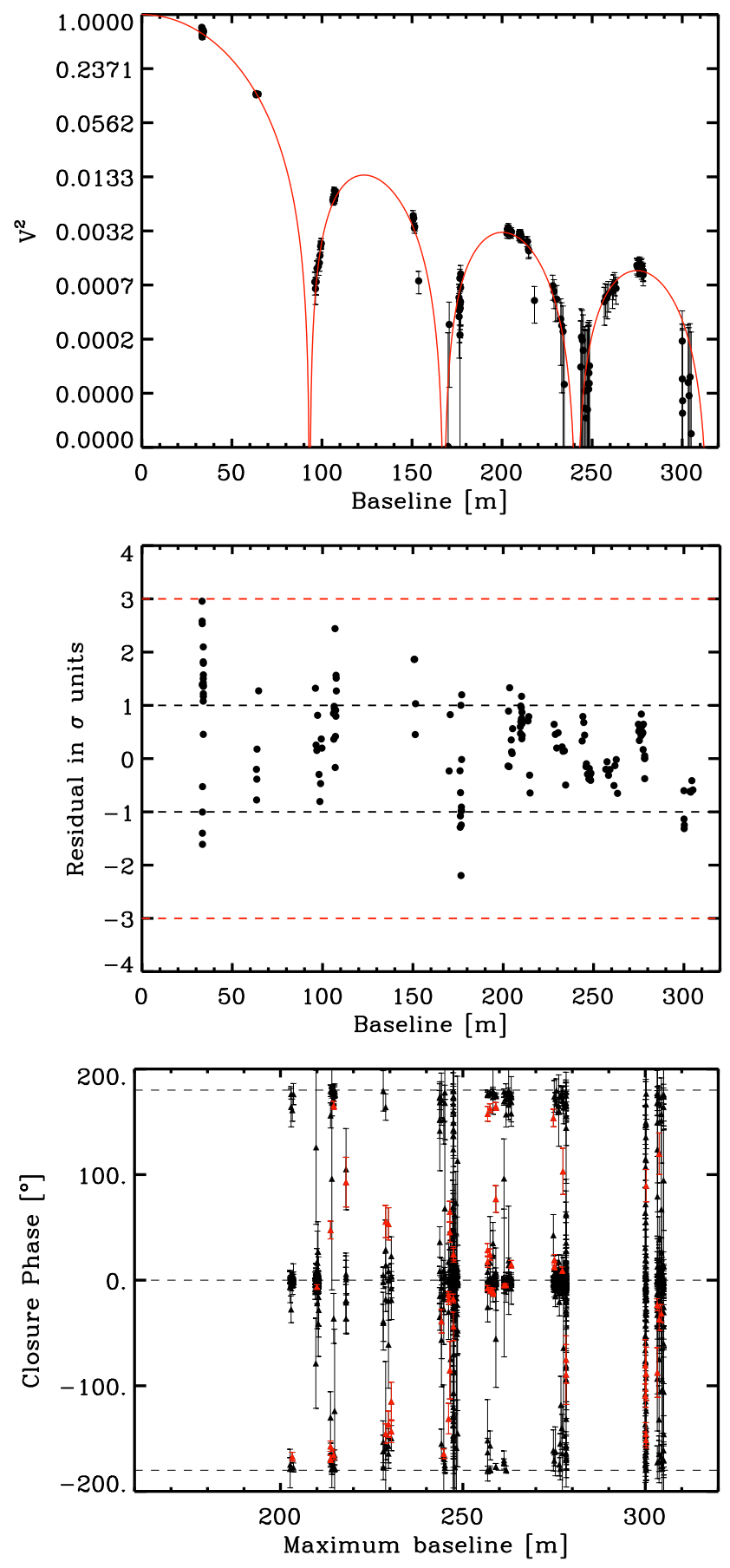

Fig. A.1. Same as in Fig. 1, but for the star $\epsilon$ Cyg.
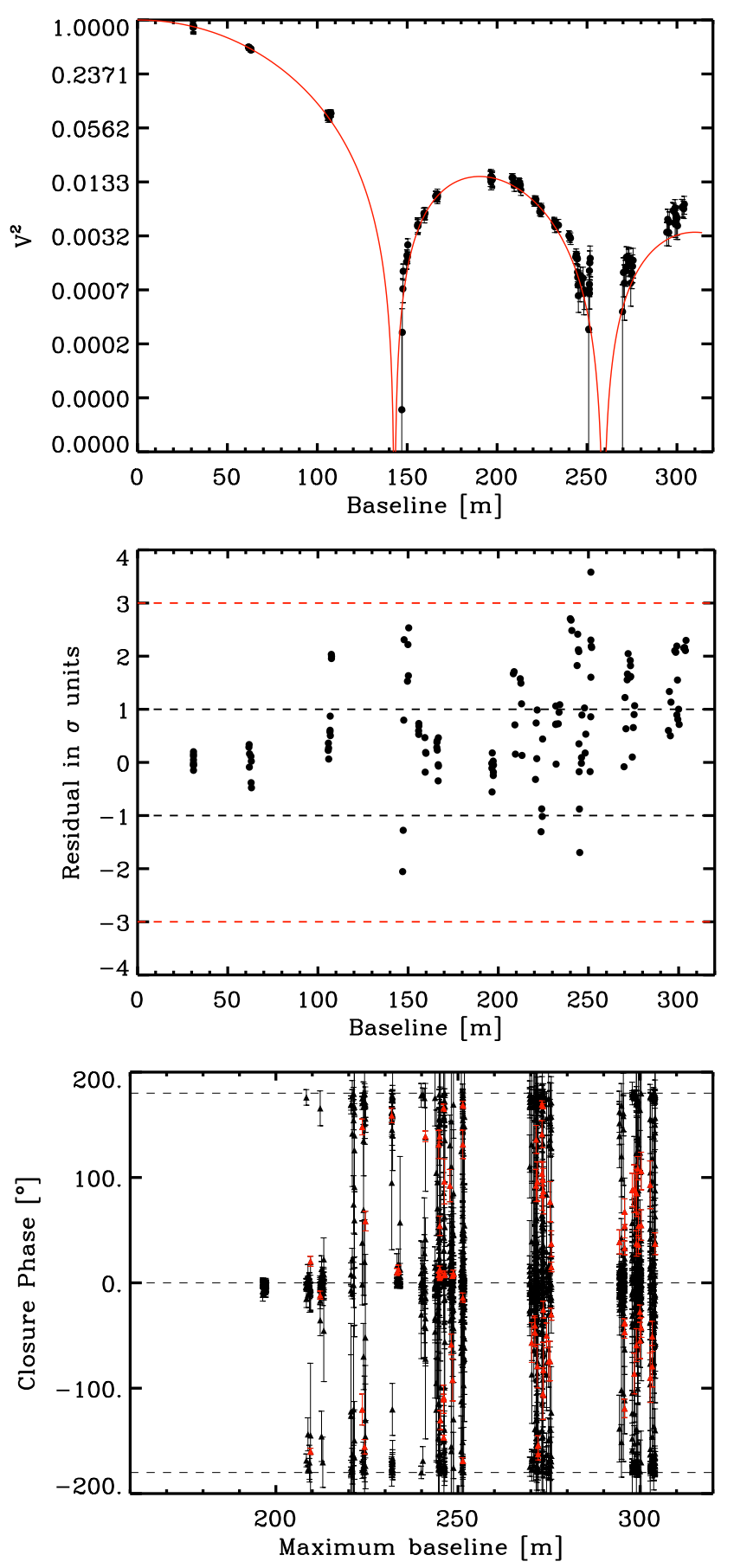

Fig. A.2. Same as in Fig. 1, but for the star HR 7633. 